\title{
Luhmann y la Complejidad: una introducción transdisciplinar
}

\section{Guilherme Brandão}

Abogado UNISANTOS (Universidad Católica de Santos), Diplomado en Administración Financiera. Estudiante del Magíster en Antropología y Desarrollo, Universidad de Chile. guisnbbrandao@gmail.com

Resumen

El artículo busca exponer la relación entre el concepto de complejidad y la producción de la estructura teórica de Niklas Luhmann, por eso, camina por la relación que existe actualmente entre las Ciencias Naturales y las Ciencias Sociales y apunta el carácter transdisciplinar del trabajo del maestro alemán, puntuando de manera más desmenuzada la importancia del concepto de autorreferencia en este proceso.

El artículo inicialmente expone la complejidad como un concepto que emerge de las Ciencias Duras y que impone a ellas gran dificultad. Este hecho indica la dificultad inherente a la manipulación de la complejidad.

Desde allí, para los científicos sociales les restaba la tarea de producir espacios teóricos que posibilitasen la manipulación de lo complejo, traducir para el lenguaje científico la contradicción tan real y concreta como lo social en si.

Luhmann puso la complejidad de las Ciencias Duras en el seno de las Ciencias Sociales, admitiendo de manera concreta que sólo la complejidad puede reducir la complejidad. La distribuyó por entre todos sus conceptos, de allí se puede decir que la complejidad modela el modelo de la teoría luhmanniana.

\section{Abstract}

The article seeks to explain the relationship between the concept of complexity and the production of the theoretical structure of Niklas Luhmann, therefore, walk by the relationship that now exists between the Natural Sciences and Social Sciences targets and the transdisciplinary nature of the work of the German master, Scoring more shredded the concept of self in this process.

The article exposes the complexity initially as a concept that emerges from the hard sciences, and it imposes on them great difficulty. This fact indicates the difficulty inherent in the handling of complexity.

From there, for social scientists have remained the task of producing spaces that permit the theoretical handling of the complex, to translate scientific language contradicts the very real and concrete as social itself.

Luhmann put the complexity of Hard Sciences within the Social Sciences, admitted in a concrete way that only the complex can reduce complexity. The distributed among all its concepts, then you can say that the complexity models the luhmannian model theory.

Palabras Clave: Luhmann, complejidad, autorreferencia, paradigma, transdiciplinaridad.

\section{Complejidad y las Ciencias Duras}

La complejidad en el principio lleva, como Morin, a lo que es irracional, incierto, confuso, desordenado. Siendo así la complejidad, es un concepto que hay que 
explorar, definir. (Morin \& Le Moigne, 2000, pp. 47). La definición de complejidad existe en muchas Ciencias, tal vez, en todas. Sin embargo, no hay acuerdo entre las definiciones resultantes, tal vez algunos puntos en común, nada más. Estos puntos se refieren a la relación entre la complejidad y sistemismo y no a la relación entre el sistema y a la complejidad (Luhmann, 1998, 53). Es útil en este contexto, tener conciencia de la profunda relación "a priori" entre la complejidad y las ciencias duras. A continuación se presentan dos ejemplos que corroboran la afirmación anterior:

"The term complexity is abundantly used in spite of the absence of a comprehensive and concise definition. Indeed the definitions employed by most researchers simply appear to reflect the particular task or model involved." (Seth, p.2)

Además, "... there is no established definition of complexity or complexity science. If you were to ask ten different people wandering the halls of the Santa Fe Institute, you'd get ten different-and likely ten very distinct-responses." (Davis et al, 2003, p.217)

Pese a la dificultad del tema la continuidad del artículo impone que se diseñe en líneas muy generales un concepto de complejidad, cuya idea busca abarcar la condición inherente del complejo y de su carácter dinámico. Por lo tanto la complejidad es un objeto que emerge en los sistemas formados por elementos interdependientes con innúmeras posibilidades de interconexión, tales sistemas intentan relacionarse de manera congruente a su entorno. Las palabras de Gershenson y Heylighen refuerzan lo anteriormente mencionado y la idea que aquí quiere registrarse, veamos:

"Complex systems will typically exhibit a tangle of interconnected positive and negative feedback loops, where the effects of any change in a component cascade through an increasing number of connected components, in part feeding back, positively and/or negatively, into the initial component. If there is a variable time delay between these effects, it becomes in principle impossible to make predictions, because we do not know who will affect who first and thus whether an effect will be dampened before it has had the chance to get amplified or not" (Gershenson y Heylighen, p. 1)

El punto crítico que se manifiesta en la idea anterior es el concepto de no-linealidad, id est, la reacción de los elementos es impredecible, contingente. No hay forma de relacionar acción y reacción, lo que hay son, infinitas posibilidades de acción y reacción, todas, a priori, igualmente válidas y posibles, inclusive las más contradictorias.

No se puede decir que la complejidad se resume a la no-linealidad - bajo ninguna hipótesis - no obstante, para los efectos de este artículo lo que se quiere exponer es que gran parte de la dificultad de tratar con el concepto de complejidad es la nolinealidad y sus consecuencias. Más allá es bastante difícil imaginar el surgimiento de la complejidad sin vínculos con la interconexión de las variables que interactúan en forma no lineal.

A continuación se profundizará el concepto de no linealidad buscando la mejor manera de exponer la dificultad inherente al manejo de la complejidad.

La no-linealidad se materializa en su casi total incapacidad de predicción. Una acción que crea cascadas de efectos en mayor o menor dimensión, que afectan mediata y/o inmediatamente un número importante - o no - de elementos interconectados por un 
sistema complejo. En su libro Caos - la creación de una nueva ciencia, James Gleick, establece un concepto muy claro de no linealidad:

"Los sistemas no lineales, en general, no se pueden resolver y no pueden ser añadido unos a los otros. En los fluidos y los sistemas mecánicos, los términos lineales tienden a ser las cosas que la gente quiere dejar de lado cuando se trata de entenderlos bien o de una manera sencilla. La fricción, por ejemplo. Sin fricción, una simple ecuación lineal expresa la cantidad de energía necesaria para acelerar un disco de caucho en un juego de hockey. Con la fricción, la relación se complica porque la cantidad de energía está cambiando, dependiendo de la rapidez con que el disco se mueve. La no linealidad significa que el acto de jugar el juego modifica, en cierto modo las reglas. No se puede asignar una importancia a la constante fricción, ya que su importancia depende de la velocidad. La velocidad, a su vez, depende de la fricción. Esta mutabilidad dependiente hace que sea difícil el cálculo de la no-linealidad" (Gleick, 1989, p. 21)

John H. Holland, en su libro "El orden oculto: como la adaptación crea la complejidad", se refiere a sistemas complejos adaptables.(SACs), es decir sistemas que tienen además de otras características la complejidad y la no-linealidad. Holland en el primer capítulo expone las dificultades que encuentra en su trabajo diario al hacer frente a la descripción de las dinámicas de los sistemas complejos:

"Nuestro objetivo consiste en aislar esos principios generales. Este objetivo es reciente, por lo que este libro no podrá hacer más que esbozar un mapa del terreno. Y gran parte de este mapa consistirá en tierras desconocidas y títulos como "aquí existen monstruos". (Holland, 1997, p. 27)

Todo lo expuesto arriba pasa a ser más y más importante desde el punto en que se nota, como explica Capra, socorriéndose en las palabras de Stewart, que "la naturaleza es inflexiblemente no lineal." Por lo tanto la linealidad, se convierte en la excepción.

Prigogine, ganador del Premio Nobel por sus esfuerzos en el estudio de los sistemas fuera de equilibrio, que básicamente son también sistemas complejos explica:

"Mi posición es que comprendemos por qué el mundo es complejo pero no aprendemos el mecanismo de la complejidad, salvo en casos muy sencillos de física o química de no equilibrio." (Prigogine in Benkirane, 2004, p. 39)

Todo lo anterior tiene como consecuencia la dificultad o imposibilidad de definir el término complejidad. Sin embargo, definida o no la complejidad, esta fue la realidad de las Ciencias Duras o Naturales desde el descubrimiento de los quanta por Max Planck. Desde aquí, con motivo de la manipulación da la complejidad pasa a existir en el seno de la Física, - cada día con mayor intensidad - la necesidad de cambio paradigmático. Ya que en cada descubrimiento se hace más concreta la probabilidad, inherente a lo no-lineal, y queda más lejana la esencia misma de las ciencias naturales: la no contradicción, la linealidad, el determinismo y la verdad absoluta, los conceptos inherentes a la fundación de Ciencias Duras o Naturales; estas son las palabras de Brian Greene:

"En 1927, la física ya había perdido la inocencia clásica. Estaban terminando los días de un universo mecánico, cuyos componentes, una vez puestos en marcha, funcionaban como un reloj, para cumplir obedientemente su destino inexorable y predeterminado. Según la mecánica cuántica, el universo no evoluciona de acuerdo con una formalización matemática rigurosa y precisa, pero que se limita a determinar

Revista Mad. № 19, Septiembre de 2008. Departamento de Antropología. Universidad de Chile http://www.revistamad.uchile.cl/19/Brandao_07.pdf 
la probabilidad de que en un futuro, en particular, pueda suceder - y no cual es el futuro en el que sucederá. "(Greene, P. 127, 2007)

El propio desenrollar de las Ciencias Duras fue desgastando sus principios, la serpiente se come su propia cola e, inevitablemente llegaría a su cabeza.

\section{Uniendo las dos puntas}

Este movimiento corría paralelo al creciente malestar de las Ciencias Sociales con sus cimientos, ya que los cambios sociales en los que se basaba el modernismo iluminaban la necesidad de hacer frente a la totalidad, allí se incluyó la contradicción, es decir, como Karel Kossik, "la totalidad sin contradicciones es vacía e inerte y las contradicciones fuera de la totalidad es abstracta si no considera simultáneamente a la base y la superestructura." Por lo tanto, la visión del materialismo dialéctico, específicamente la de Engels, bastante relegada por la corriente principal de Ciencias Sociales, daría el tono futuro de las Ciencias Sociales. Reabriría puertas (teniendo en cuenta el pensamiento de Heráclito) nuevamente para la manipulación de lo contradictorio y lo incierto.

Cabe destacar aquí que tal punto de vista, en Física Cuántica por ejemplo, pasaba a emerger como conclusión de los últimos acontecimientos, aquella misma que porfiaba querer tirar abajo la construcción racional, y en este punto se encuentra con las perspectivas de Heráclito, Pascal, Engels. Pensadores que habían expuesto un pensamiento, resumidamente contrario a la no-contradicción aristotélica y favorables a las contradicciones inherentes a la totalidad. El pensamiento de Engels muy bien ilustra el tema y su alcance en relación al "corpus" científico y, por lo tanto, más allá de la distinción Ciencias naturales o sociales, véase:

"Thus we have once again returned to the point of view of the great founders of Greek philosophy, the view that the whole of nature, from the smallest element to the greatest, from grains of sand to suns, from protista to men, has its existence in eternal coming into being and passing away, in ceaseless flux, in un-resting motion and change, only with the essential difference that what for the Greeks was a brilliant intuition, is in our case the result of strictly scientific research in accordance with experience, and hence also it emerges in a much more definite and clear form. " (Engels, versión electrónica, p.12)

O no...

\section{Ciencias Sociales}

Las ciencias sociales nacieron bajo la égida del positivismo que parte de las premisas científicas fundadas por los pensadores griegos, perfeccionadas en el seno del racionalismo cartesiano, y puestas en práctica en la Física de Newton, en el determinismo de La Place y el empirismo de Bacon. De este modo, la Ciencias Sociales han manipulado, hasta que se demuestre lo contrario, su objeto de estudio de manera no contradictoria, lineal y reduccionista.

Los positivistas vinculaban el conocimiento a algo observable, hágase saber, la validez de una ley estaba directamente relacionada a lo palpable, observable, medible, salvaguardando, de esta forma, la objetividad de la ciencia. Por lo tanto, inexistiendo la posibilidad de observación calcada en parámetros rigurosos no existiría la producción de conocimientos científicos. 
El positivismo predicaba una ciencia objetiva, neutra, a-histórica, capaz de formular leyes generales sobre el funcionamiento de la naturaleza, y más:

"..... los positivistas no estaban interesados exactamente en como los científicos pensaban, en sus motivaciones o como ellos actuaban en la práctica: esto sería una tarea para la psicología y la sociología. Lo que interesaba eran las relaciones lógicas entre los enunciados científicos. La lógica de la ciencia, proveía un criterio ideal de como el científico o la comunidad científica debía reaccionar o pensar, teniendo, por lo tanto, un carácter normativo en vez de descriptivo. El objetivo central no era el de explicar como la ciencia funciona pero sí, justificar o legitimizar el conocimiento científico estableciendo sus fundamentos lógicos y empíricos. "(Alves -Mazzoti y Gewandsznajder, p. 13).

De este modo, el escenario producido por el paradigma positivista, como se podría imaginar no tenía posibilidad de acomodar confortablemente a las ciencias sociales.

Las interrelaciones sociales no posibilitan generalizaciones reduccionistas, no evolucionan de forma lineal, determinista, a pesar de la intención físico matemática buscada por Durkheim, sin embargo bajo un trasfondo organicista. Lo social que se quería considerar orgánico, por lo tanto, un todo formado por partes que mantenían relaciones interdependientes se reducía a un objeto que se relacionaba de forma lineal, resumiéndose a leyes de causa y efecto.

Esto se debe porque, a pesar de la sistematicidad estar vinculada al núcleo del concepto de la ciencia desde Aristóteles (así, la Ciencia - para ser Ciencia - debería y debe aún hoy, basarse en la organización de su corpus, en la interconexión de los conocimientos extraídos mediante la utilización de sus métodos) el uso de métodos científicos con el fin de producir conocimiento contextual, holístico de su objeto de estudio, se destaca, la extracción de conclusiones científicas, no por medio del estudio de sus partes de manera aislada, sino como un todo, en su totalidad, demoró siglos para desarrollarse. Por lo tanto, la transposición de la sistemacidad inmanente del concepto de Ciencia para su práctica efectiva, mejor diciendo, para el objeto de estudio de esta misma ciencia, demoró el tiempo que se extiende desde la Grecia antigua hasta los días de hoy. Esta incapacidad para la contextualización afecta más frontalmente, a priori, a las ciencias sociales.

Con respecto al lugar del hombre en la ciencia, así expone Foucault:

"La primera cosa para constatar es que las Ciencias Humanas recibieron por herencia un determinada un determinado dominio ya delineado, dimensionado tal vez en su conjunto, pero no desbravado, y que ellas tendrían por tareas desarrollar como conceptos científicos y métodos positivos; el siglo XVII no les transmitió, bajo el nombre de hombre o de naturaleza humana, un espacio circunscripto exteriormente, pero todavía vacío, para que ellas tuviesen en seguida, la tarea de cubrir y analizar.

El campo epistemológico que recorren las Ciencias Humanas no fue prescripto de antemano: ninguna filosofía, ninguna opción política o moral, ninguna ciencia empirica, cualesquiera que fuese, ninguna observación del cuerpo humano, ningún análisis de la sensación, de la imaginación, o de las pasiones jamás la se encontró; en los siglos XVII y XVIII alguna cosa como el hombre, pues, el hombre no existía ( así como la vida, el lenguaje y el trabajo); y las Ciencias Humanas no aparecieron cuando, bajo el efecto de algún racionalismo urgente, de algún problema científico no resuelto,

Revista Mad. № 19, Septiembre de 2008. Departamento de Antropología. Universidad de Chile 
de algún interes práctico, se decidió, hacer pasar al hombre ( por bien o por mal y como mayor o menor éxito) para el campo de los objetos científicos - en cuyo número tal ves no esté, todavía probado que sea posible incluirlo de un modo absoluto; ellas aparecieron el día en que el hombre se constituyo en la cultura occidental, al mismo tiempo como lo que es necesario y lo que se debe saber. "(Michel Foucault, pp 475, 476)

Desmenuzando las palabras de Foucault, se observa que en el caso de las Ciencias del hombre, el camino tendría que ser desbravado, una nueva página de la historia debería ser escrita, apenas se empieza la redacción se descubre entre innúmeras otras cosas, que el científico social se confunde con el objeto analizado restando imposible proteger a la objetividad exigida por los positivistas.

Dada la situación, suele decirse que la estructura que abarcaba las Ciencias Sociales no la confortaba, no cubriría a su sujeto, además, no abarcaría la relación existente entre sujeto y objeto, ya que observador y objeto observado se confundían y continúan confundiéndose. Visto de esta manera, en lo que se refiere a las interrelaciones del hombre en el seno de la sociedad no hay objetividad, no hay claridad, hay contradicciones, la incertidumbre, la complejidad del ser humano en todas sus gamas.

Sin embargo, el siglo $X X$, reveló sorpresas para la racionalidad que dominó la ciencia clásica conforme concebida por Newton, de modo que hubo un profundo estremecimiento en el andamiaje científico natural, ipso facto, las Ciencias del Hombre vienen acercándose de dichas Ciencias Duras o Naturales, estas son las palabras de Boaventura Santos:

"...las ciencias naturales son todavía hoy diferentes de las ciencias sociales pero se aproximan cada vez más de ellas y es posible que en un futuro no muy lejano, se diluyan en ellas, por dos razones teórico prácticas. En primer lugar, el progreso científico de las ciencias naturales es el principal responsable por la crisis del modelo positivista y, por causa de ella, las características, que antes dictaron la precariedad del estatuto epistemológico de las ciencias sociales, son reconceptualizadas y pasan a apuntar al horizonte epistemológico posible para las Ciencias en un todo. En segundo lugar la materialidad de la tecnología en que el avance científico de las Ciencias Naturales se estampó no hizo con que los objetos teóricos de las Ciencias Naturales y de las Ciencias Sociales dejasen de ser distintas, pero hizo con que aquello en que son distintas, sea progresivamente menos importante de que aquello en que son iguales. "(Santos, 2000, p.52)

La igualdad, in casu, se refiere a la inherencia del trato con la probabilidad, con el contingente, con la no-linealidad, lo complejo en sí. La unión de los diversos campos científicos se produce en el seno de la complejidad y sólo a través de un profundo entrelazamiento de las diversas ciencias pasa a ser factible su manipulación. La complejidad exige no la interdisciplinaridad, no la multidiciplinarid, exige si la transdiciplinaridad, siempre. Importante decir, entrelazamiento que termina con la diferenciación hasta donde es posible entre el social y el natural, el hard de las Naturales y el soft de las Sociales, dejan de tener la importancia que un día tuvieron.

Por lo tanto, para una andamiaje que amparase las Ciencias Sociales suficiente era encontrar una manera de exponer la no linealidad en su seno. Bastaba dominar la complejidad social. En fin, tarea muy difícil. 
Para los científicos sociales les restaba la tarea de producir espacios teóricos que posibilitasen la manipulación de lo complejo, llevar a cabo la linealidad, terminar con el reduccionismo estéril, con la vinculación entre causa y efecto, exponer el no-ser como posibilidad, traducir para el lenguaje científico esta contradicción tan real y concreto como lo social en si.

Este, es el punto de partida de la idea de estructura general para una teoría de la sociedad moderna del alemán Niklas Luhmann. Veamos:

"Si se quiere que se la considere como parte de una ciencia de la realidad, esclarecedora del mundo, la sociología deberá ubicar el problema de la complejidad social en el centro de su interés." (Luhmann, 1973, p.107)

Entonces, para Luhmann la relación entre Ciencias Sociales/Ciencias Naturales se exponía de manera muy parecida a de Santos. Los dos grandes senderos tienen hoy, corroborando con lo anteriormente mencionado, un gran punto en común, veamos:

"Esta división de los métodos indica el problema central de los grupos de conocimiento y de los dos tipos diferentes de investigación. Dichos grupos se perciben, todavía hoy, como problemas aparentemente irresolubles que ya no se identificaban con una materia específica o dominio de indagación, sino más bien con el problema de la complejidad para las ciencias, y del sentido para las humanidades."

En esta discusión, sin embargo, se echa de menos ahondar el paralelismo que existe entre el concepto de sentido y el de complejidad. El sentido es una representación de la complejidad." (Nafarrate, 1996, p.178)

\section{Los malestares iniciales}

La paradoja, lo improbable, el "paso en la oscuridad" no afectó a Luhmann como hizo un día con aquellos que sacudieron los cimientos de la ciencia dura (Max Planck, Einstein). Tales científicos, que a pesar de pruebas claras, no se sentían cómodos en ir más allá de la estructura clásica y racional, a pesar de que la producción de sus teorías probaba lo contrario. El contexto, entonces, revelaba la contradicción.

Planck nueve años después de la construcción de su constante, continuaba, contradictoriamente, a porfiar con ella, su propia creación, y tener explosiones como defensor del viejo orden lo que también pasaría con Einstein, veamos: "Tengo muchas esperanzas de resolver el problema de la radiación, y que puede hacerlo sin los quanta de luz. " De esta manera, buscaba mantener firme la ilusión del poder inquebrantable de la estructura clásica newtoniana, ja incapaz de sustentarse frente a lo nuevo e in sustentable por el trabajo de los dos arriba citados y, a los cuales se sumarán otros (a favor de una postura novedosa en las Ciencias Duras, se debe citar, Niels Bohr, el padre del modelo atómico y Werner Heisenberg, el padre de la teoría de la Incertidumbre, epistemologicamente, una de las condiciones de posibilidad de la teoría luhmanniana)

Luhmann, a su vez, convivía bien con la imposibilidad de que tenían los clásicos para explicar la realidad social del siglo XX. Sin embargo, la recepción de su obra, en principio fue contradictorio, el rechazo convivía con ávidas defensas (Izuzquiza, 27), tal vez el mismo escenario de las Ciencias Duras, pero, presentando otros matices - y aún hoy existe la misma situación conforme las palabras de Nafarrate en la introducción del libro Sistemas Sociales de Niklas Luhmann. La necesidad de defender

Revista Mad. № 19, Septiembre de 2008. Departamento de Antropología. Universidad de Chile http://www.revistamad.uchile.cl/19/Brandao_07.pdf 
nuevos caminos caía muy bien, consciente que era del hundimiento de los clásicos, su "déficit teórico" para lidiar con la modernidad (Weaver, 1947), así:

"Concluyamos pues que Luhmann construye su teoría desde una crítica abrumadora contra las grandes luminarias de la tradición sociológica." (Izuzquiza, 54)

Tales consideraciones tienen que ser citadas apenas para poner luz a la grandiosidad de las alteraciones científicas del siglo XX o cuanto se tuvo que alterar la propia epistemología de la ciencia para abarcar la ""realidad"" absolutamente nueva que se descortinaba y la necesidad de, por un lado a otro los diversos campos científicos, como por ejemplo buscó hacer Bertallanfy

\section{Antes de 1984 ..... pero, con solidez}

Luhmann, en sus primeros escritos, hace hincapié en la necesidad de exponer críticamente la racionalidad teleológica, recolocándola en la teoría de los sistemas. La teoría de la acción, históricamente considerada en el seno del esquema ontológico griego como causa y efecto, sería reinterpretada en base sistémica. Por lo tanto desarrolla críticamente la relación entre fines y medios, partiendo de los nuevos enfoques de la teoría social, de la teoría de la decisión, la teoría de las organizaciones, de la Cibernética. Así, uno puede explicar los primeros pasos de la teoría luhmanniana:

"De esa manera aflora una dimensión enteramente nueva de la complejidad. La perdida de la verdad teleológica común, la subjetivización del establecimiento de los fines hace consciente al otro hombre en su condición de libre alter ego en la historia europea las guerras civiles de religión del siglo XVI contribuyeron lo suyo a la ilustración de este problema. Con esta nueva complejidad se transforma el sentido de la racionalidad en un modo apenas percibido. Ya no puede entenderse la racionalidad como despliegue inteligente y como observancia de un sentido previamente dado. Es, por encima de toda reducción de complejidad.

Estas consideraciones remiten al sistema de sujetos, que aparece implícito en la representación de un compromiso basado en fines y al que ha de hacerse, ahora más explícitamente, tema de reflexión. Para ello habría que transformar el concepto de lo racional de una simple racionalidad de acción, teleologicamente orientada en una más compleja y comprensiva racionalidad sistémica. Su sentido resultaría de la referencia al problema de la complejidad. Una reorientación de esta índole viene suficientemente preparada, como hemos de ver, gracias a lo más recientes desarrollos que han experimentado diversas ciencias empíricas." (Luhmann, 1973,p. 17).

De las palabras anteriormente mencionadas se constata la construcción de una teoría que tiene como propósito remontar la racionalidad de la acción, una vez ontológica, en racionalidad sistémica; la racionalidad lineal, vendría a ser probabilidad de razón, razón no-lineal, tan cierta como incierta, inherente al vivir en sociedad

El maestro alemán, con el objetivo de flexibilizar aún más su estructura teórica la expone bajo una base funcionalista, aún sobre el concepto de función extraído de las Matemáticas, no aquel que fue desarrollado por Parsons. En el que a pesar de partir del andamio parsoniano, Luhmann desarrolla un concepto de función, apoyado en planteamientos de Kant, basándose, repito en fundamentos matemáticos, véase:

"Una función es por lo tanto - en un todo de acuerdo con la definición de Kant - " la unidad de la acción de ordenar diversas ideas bajo otra común. Este concepto de 
función sirve en última instancia de fundamento a la teoría lógica y matemática de la función." (Luhmann, 1973, p. 20)

Sobre la base del marco funcionalista la crítica a la causalidad racional se vuelve más potente, de hecho, la función introducida por Luhmann, tenía como base exactamente poner en segundo plano el lineal, o abrir más posibilidades de contextualización, permitiendo lo no lineal, la contingencia. Buscando mejorar, en definitiva, el poder de selección, veamos: "el método funcionalista debe fundamentar precisamente la posibilidad de que algo puede ser y también no ser, y que algo es reemplazable." (Luhmann, 1973, p.23)

Luhmann, por lo tanto, va produciendo una estructura altamente flexible con la intención de traer para el pensamiento social espacio para manosear la no-linealidad y la complejidad. Sus opciones a lo largo de su obra refuerzan estas palabras.

Uno de los pilares de la teoría luhmanniana es el concepto de sistema. Para Luhmann este concepto se construye por la diferencia. Sistema es la unidad de la diferencia entre él mismo y el entorno donde está contenido, dice respecto, a graduaciones de complejidad. Siendo que la complejidad del entorno, pre-condición de la existencia del sistema, es más grande que la complejidad del sistema.

Dentro del concepto del sistema, Luhmann expone todo lo social, la sociedad mundial, desde el sistema más simple (interacción) al más complejo (social), pero no reductibles entre sí, une, de esta manera, en un solo marco teórico lo máximo y lo mínimo.

Luhmann al conceptuar la sociedad deja de lado el modelo de sistema mecanicista y la vieja tradición clásica, donde sociedad se une al concepto de autarquía, capaz de producir concepto que abarca una mayor complejidad. Por consiguiente, diferentes funciones y un mayor número de estados posibles. De este modo, la sociedad en si no controla, no impone, no toma partido ya sea para el lado político o económico.

El sistema social total, la sociedad mundial, no tiene límites terrestres, y está formado por subsistemas que allí están para reducir la complejidad, imposible de ser totalmente manipulada. El sistema social es condición de existencia de tantos otros subsistemas y estos son prolongamientos de la capacidad selectiva del sistema social causando el flujo incesante, interdependiente y siempre contextual de la reducción y aumento de la complejidad.

\section{Después de 84}

Luhmann en el año 1984, edita el libro "Sistemas Sociales". Según la tradición de la Ciencias Duras, expone definición operativa de complejidad, veamos:

"..la complejidad es talvez el punto de vista que expresa con más fuerza las experiencias problemáticas de la investigación moderna de sistemas. En esas experiencias ha asumido la función catalizadora, sin que se haya llegado a definirla. Esto dificulta, sin embargo, un trabajo de mayor control con el concepto. Sin dejar de tomar puntos de referencia en la bibliografía especializada, elegimos un concepto orientado al problema y lo definimos sobre la base de los conceptos de elemento y de relación." (Luhmann, 1998, p.47) 
Además, en el libro, Luhmann toma, tal vez una de las decisiones más importantes de su obra uniendo la comunicación y la acción, exponiendo aquella como el elemento constitutivo de la sociedad, abriendo así, espacio en su andamiaje para la autorreferencia. ¿Cuál es la lógica detrás de esto? Modelar en la teoría de los sistemas la complejidad del mundo, veamos:

"La autorreferencia es un correlato de la presión de la complejidad universal. En ninguna parte del mundo su complejidad puede ser reproducida, reelaborada, controlada adecuadamente, porque entonces aumentaría en la misma proporción. En su lugar se crea la autorreferencia que puede ser reespecificada para enfrentarse a la complejidad" (Luhmann, 1998, p.396)

El auto referencia pasa a ser parte de la teoría Luhmanniana después de largo proceso. A pesar de Luhmann, flirtear con ella por la belleza de las respuestas presentadas por la aplicación de la fenomenología transcendental de Husserl, su integración ocurre después de la obra de Maturana Y Varela y más significativamente después de Luhmann, estar seguro de tener el elemento perfecto (conforme su teoría) para ser producto, productor y reproductor de la complejidad social: la comunicación.

Pero, ¿cual es la ventaja del modelar a través de la autorreferencia y en que medida tal proceso ayuda la Ciencia? Luhmann, en ese caso opta por la mejor descripción, la mejor "comprensión y reducción de la complejidad universal mediante la formación de sistemas" (Luhmann, 1973,p. 119) autorreferenciales.

El anteriormente mencionado se contrasta con la seguridad de Luhmann y con su teoría más madura así expone en Sistemas Sociales, véase:

".....ahora hay que preguntarse por las causas de las causas. Por ello, el regreso ad infinitum es equipado con esperanzas de aproximación que encuentran su seguridad última en el funcionamiento de la complejidad. Si las causas se fundamentan de nuevo y si cada etapa se mantiene abierta a la crítica y dispuesta a la revisión, resulta cada vez más improbable que una edificación así realizada pudiera presentarse sin ninguna referencia a la realidad. La circularidad no se ha eliminado, se le ha utilizado, desplegado y destautologizado. Sin esta autorreferencia basal, cualquier conocimiento se derrumbaría. Sólo por medio de ella es posible representar una estructura sensible al entorno que obtenga información de lo que la ciencia llama realidad (asuntos, objetos)" (Luhmann, 1998, p.425)

La autorreferencia para Luhmann es, por lo tanto, una herramienta fundamental a la producción de informaciones a respecto de la complejidad social. El no uso de tal herramienta, conforme el pensamiento de Luhmann, imposibilita la operación, imposibilita la propia Ciencia, ya que, la concepción y reducción de la complejidad, impone creciente carácter autocrítico a estructura científica en si.

\section{Conclusión}

Luhmann puso la complejidad de las Ciencias Duras en el seno de las Ciencias Sociales, admitiendo de manera concreta que "sólo la complejidad puede reducir la complejidad." (Luhmann, 1998, p.49) La distribuyo por entre todos sus conceptos, de allí se puede decir que la complejidad modela el modelo de la teoría luhmanniana y así emerge en el medio de las observaciones producidas, imponiendo que siempre podría ser de otra manera, haciendo la teoría cuestionar la propia teoría en cada proceso. Y, por lo tanto, haciendo con que la no-linealidad, al envés de enemiga, fuera más un 
elemento existente para la mejor descripción, elemento que genera una mayor problematización a partir de la observación. Elemento, lo cual, produce mayores posibilidades de selección.

Luhmann manipuló la complejidad dura que desafió y desafía aún las Ciencias Duras, la transformó en objeto de estudio social, exigió, conciente o inconcientemente, la exigencia que es inherente de la complejidad, cual sea, la transdiciplinaridad. Admitiendo acá como transdiciplinaridad, lo que emerge en el encuentro de innumerables disciplinas, por lo tanto, un otro andamio teórico de donde nacen conceptos que, repito, emergen de la interrelación de sus partes. Así el nuevo que no se define por interdisciplinaridad, multidiciplinaridad, o pluridiciplinaridad, objeto que hace con que tales conceptos no más tengan fuerza explicativa.

Así, se puede decir con seguridad que la teoría luhmanniana es el entrelazamiento de innumerables teorías de donde nace otra teoría, nueva, novedosa, flexible, que se crítica al largo de todo el sendero, no perfecta, que no carga consigo ninguna verdad absoluta. Tal vez se pueda decir que hay un paradigma cristalizado alrededor de la teoría luhmanniana..... no ? En definitiva, se puede decir que Niklas Luhmann alzó para otro estadio el pensamiento sistémico en las Ciencias Sociales. RM

\section{Bibliografía}

ALVES-MAZZOTTI Alda J. \& GEWANDSZNAJDER Fernando. O método nas Ciências Naturais e Sociais: pesquisa quantitativa e qualitativa. São Paulo - SP. Editora Pioneira. 2001

BENKIRANE, Redá. "A complexidade, vertigens e promessas - Histórias de Ciências" ; tradução de Maria João Batalha Reis. Lisboa, Portugal. Instituto Piaget. 2004.

CAPRA, Fritjof.. "A rede da vida: uma nova compreensão científica dos seres vivos." ; tradução de Newton Roberval Eichemberg. 16 Edição. São Paulo. Editora Cultrix, 2000.

CATHALIFAUD, Marcelo A. \& MANSILLA, Dario R. "Sociedad y teoría de sistemas". 4 edición, Santiago de Chile. Editorial Universitária, S.A., 2007

DAVIS,Brian et al. "Complicity : an introduction and a welcome'

Internet:

http://www.complexityandeducation.ualberta.ca/COMPLICITY1/pdfs/Complicity11b_Int ro.pdf

ENGELS, Frederick. "Dialectics of Nature". E-version

FOUCAULT, Michel. "As palavras e as coisas: uma arqueologia das Ciências Humanas"; tradução Selma Tannus Muchail. 8 o Edição. São Paulo: Martins Fontes, 1999.

GERSHENSON Carlos \& HEYLIGHEN, Francis. "How can we think the complex". Internet: http://arxiv.org/ftp/nlin/papers/0402/0402023.pdf

GLEICK, James. "Caos: a criação de uma nova ciência" ; tradução de Waltensir Dutra. $15^{\circ}$ Edição. Rio de Janeiro. Elsevier. 1989

Revista Mad. № 19, Septiembre de 2008. Departamento de Antropología. Universidad de Chile http://www.revistamad.uchile.cl/19/Brandao_07.pdf 
HOLLAND, Jonh H. "A ordem oculta - como a adaptação gera a complexidade" ; tradução de José Luis Malaquias. Lisboa, Portugal. Gradiva Publicações Ltda. 1997.

Izuzquiza, Ignácio. La sociedad sin hombres: Niklas Luhmann o la teoría como escandalo. Barcelona, España. Editorial Anthropos. 1990

LE MOIGNE, Jean-Louis. "A Teoria do Sistema Geral: Teoria da modelização" ; tradução de Jorge Pinheiro. Lisboa, Portugal. Instituto Piaget. 1996.

LUHMANN, Niklas. "Ilustración Sociológica y otros ensayos". Buenos Aires, Argentina Editorial Sur.. 1973

LUHMANN, Niklas. Sistemas Sociales: Lineamientos para una teoría general. Barcelona, España. Anthropos Editorial. 1998

MORIN, Edgar \& LE MOIGNE, Jean Louis. "A inteligência da complexidade" ; tradução de Nurimar Maria Falci. $3^{\circ}$ Edição. São Paulo: Peirópolis. Editora Fundação Peirópolis Ltda. 2004.

NAFARRATE, Javier T. "Introducción a la teoría de Sistemas". Mexico, DF. Anthropos Editorial. 1996

NUSSENZVEIG, H Moysés. "Complexidade e Caos". 20 Edição. Rio de Janeiro. Editora UFRJ/ COPEA. 2003

SANTOS, Boaventura Sousa. "Introdução a uma Ciência Pós-Moderna". São Paulo. SP. 3 edição. 2000

SETH, Anil K. Interaction, "Uncertainty and the evolution of complexity".

PASCAL, Blaise. Pensamentos. São Paulo. Ed. Martins Fontes. 2005

VASCONCELLOS, Maria José Esteves de. "Pensamento Sistêmico o novo paradigma da ciência" $4^{\circ}$ Edição. Campinas. São Paulo. Papirus Editora. 2005

WEAVER, Warren. "Science and Complexity". Internet:

http://www.ceptualinstitute.com/genre/weaver/weaver-1947b.htm 